\title{
Mapping kinematic functional abilities of the hand to three dimensional shapes for inclusive design
}

\author{
Samuel T Leitkam ${ }^{\mathrm{a}}$, Laura Bix ${ }^{\mathrm{b}}$, Javier de la Fuente ${ }^{\mathrm{c}}$, Tamara Reid Bush ${ }^{\mathrm{a}, *}$ \\ a Department of Mechanical Engineering, Michigan State University, East Lansing, MI 48824, USA \\ b School of Packaging, Michigan State University, East Lansing, MI 48824, USA \\ c Industrial Technology, California Polytechnic State University, San Luis Obispo, CA 93407, USA
}

\begin{abstract}
A B S T R A C T
Loss of hand function can have adverse effects on an individual's ability to maintain independence. The ability to perform daily activities, such as food preparation and medication delivery, is dependent on the hand's ability to grasp and manipulate objects. Therefore, the goal of this research was to demonstrate that three dimensional (3D) modeling of hand function can be used to improve the accessibility of handheld objects for individuals with reduced functionality through informed design. Individual models of hand functionality were created for 43 participants and group models were developed for groups of individuals without (Healthy) and with reduced functionality due to arthritis (RFA) of the hand. Cylindrical models representative of auto-injectors of varying diameters were analyzed in 3D space relative to hand function. The individual model mappings showed the cylinder diameter with the highest mapped functional values varied depending on the type of functional weighting chosen: kinematic redundancy of fingertip pad positional placement, fingertip pad orientation, or finger force directionality. The group mappings showed that for a cylinder to be grasped in a power grasp by at least $75 \%$ of the Healthy or RFA groups, a diameter of $40 \mathrm{~mm}$ was required. This research utilizes a new hand model to objectively compare design parameters across three different kinematic factors of hand function and across groups with different functional abilities. The ability to conduct these comparisons enables the creation of designs that are universal to all - including accommodation of individuals with limits in their functional abilities.
\end{abstract}

\section{Introduction}

The capacity to perform many of the activities during daily living is dependent on the hand's ability to grasp and manipulate handheld objects. However, increased age has been shown to correlate with losses in the ability to use the hand (Ranganathan et al., 2001). In addition, ailments such as arthritis, stroke, carpal tunnel syndrome, and hand injury adversely affect hand function for millions of individuals (Helmick et al., 2008; Jackson, 2001; Luckhaupt and Dahlhamer, 2013; Roger et al., 2012). These conditions result in decreases in joint range of motion (ROM) and in the ability to generate forces with the hand (Carmeli et al., 2003). These decreases have been shown to lead to limited capacity to perform activities of daily living (Dellhag and Bjelle, 1999) and, consequently, loss of independence (Covinsky et al., 2008). In order to maintain the ability to manipulate objects as functional capability is lost, either the individual needs to adapt and

\footnotetext{
* Correspondence to: Michigan State University, Department of Mechanical Engineering, 428 S, Shaw Lane, Rm 2555 Engineering Building, East Lansing, Ml 48824-1226, USA. Tel.: +1 517353 9544; fax: +1 5173531750.

E-mail address: reidtama@msu.edu (T. Reid Bush).
}

use different strategies, or the object being manipulated must be designed to match the abilities of the person. As such, there is a need to understand interactions between the hand and the object.

Finger motions and force generations have been studied in depth as a means to understanding human motor control. Because the hand has so many possible degrees of freedom to control and it uses them to accomplish such a broad range of tasks, understanding how the hand completes each task is not trivial. Concepts such as finger synergies (Latash, 2010; Latash et al., 2007; Visser et al., 2002) and enslaving (Kim et al., 2008; Zatsiorsky et al., 1998) have been studied in detail as ways of explaining how the human body simplifies the complex tasks of controlling force production of a kinematically and kinetically redundant system. While these studies and many more advance the understanding of the control of the human hand, they lack direct transferability to 3D spaces in a way that can be used to inform object design.

Several models define the abilities of the hand in terms of reachable 3D spaces and have the potential to quantify handobject interactions (Dias et al., 2009; Dragulescu et al., 2007; Johnson et al., 2010; Kuo et al., 2009). The weighted fingertip space (WFS) model developed by the authors is one such model that 
calculates the reachable spaces and weights the spaces according to kinematic functional abilities (Leitkam et al., 2013). The WFS model defines 3D reachable hand spaces using hand anthropometry and joint angles as input and then weights the reachable points to identify the (1) relative number of possible finger postures that allow a fingertip to reach each point, (2) range of possible orientations that the fingertip could assume at each point and (3) angular range of possible directions the fingertip could apply forces at each point. The WFS model has been shown to successfully quantify hand functionality for populations of individuals with and without reduced functionality of the hand (Leitkam and Bush, 2015). While these fingertip workspace models exist and have the potential to translate the 3D kinematic abilities of the hand into object design spaces, none have yet done so.

Accordingly, the goal of this research was to demonstrate that the WFS model can be used to determine the shape of a handheld object that best matches the kinematic functional abilities of individuals, including those with reduced hand function. Matching the object design to the hand's capabilities will lead to improved product designs so that individuals with reduced function can manipulate objects needed for everyday life and maintain independence.

\section{Methods}

\subsection{Participants}

Two groups of individuals were included in this research. The Healthy group consisted of 10 women and 12 men, ages 18-39 (mean 25.6, SD 5.8) without any reported injury or difficulty using their hands. The group with reduced functionality due to arthritis (RFA) consisted of 16 women and 5 men that were over the age of 65 (mean 72.6, SD 5.9) with self-reported cases of doctor-diagnosed arthritis. The Healthy group had an average hand breadth of $82.98 \mathrm{~mm}$ (SD $6.96 \mathrm{~mm}$ ) and hand length of $181.58 \mathrm{~mm}$ (SD $17.71 \mathrm{~mm}$ ), and the RFA group had an average hand breadth of $83.52 \mathrm{~mm}$ (SD $5.64 \mathrm{~mm}$ ) and hand length of $184.80 \mathrm{~mm}$ (SD $13.42 \mathrm{~mm}$ ). Hand sizes for both the Healthy and RFA groups ranged from at least 25th to 90th percentile based on both hand breadth and hand length for both males and females (Greiner, 1991). Informed consent was obtained from each participant under the direction of Michigan State University's Institutional Review Board (IRB \# 09-179).

\subsection{WFS modeling}

The framework for evaluating and modeling hand capabilities was the WFS model, previously developed by the authors (Leitkam et al., 2013). The WFS model was a 3D volume representing points that were reachable by each fingertip pad in space and weighted by three parameters that addressed levels of functionality for the fingertips.

1. The Redundancy weighting represented the kinematic redundancy of the hand as measured by the relative number of possible finger postures that allow a fingertip to reach each point.

2. The Orientation weighting represented the angular range of possible orientations that the fingertip could assume at each point.

3. The range of force application directions, or FAD weighting, represented the angular range of possible directions the fingertip could apply forces at each point.

\subsection{Development of individual WFS models}

The WFS model for each individual was developed by modeling the hand as a system of 16 different rigid bodies, corresponding to each of the phalanges of the hand, the first metacarpal and the palm. The rigid bodies were connected with 15 different joints, capable of producing 20 unique angular rotations, corresponding to flexions/extensions and abductions/adductions of the fingers, and flexions/extensions and rotations of the thumb about the carpometacarpal joint. The lengths of each of the bodies were measured for every individual using a caliper. The ROM for each angular rotation was determined using motion capture measurements. Detailed descriptions of the specific motions, hand measurements and targeting protocol can be found in previously published work on the development of the WFS model (Leitkam and Bush, 2015; Leitkam et al., 2013).

The rigid body model of the hand was then used to calculate equations for the fingertip position and orientation for each finger with respect to the palm as functions of the joint angles using the Denavit-Hartenberg convention. Fingertip positions, orientation vectors, and possible force application direction vectors were calculated for all joint angle combinations feasible within each finger's ROMs. The fingertip position was defined as the center of the palmar surface of the distal phalange, halfway between the DIP joint and the fingertip along the centerline of the finger. The orientation was defined with unit vectors normal to the center of the palmar surface of the distal phalange. The FADs were unit vectors originating at the center of the palmar surface and pointing in the direction of the gradient of motion of each of the flexion joints of the finger. By rounding the position coordinates of the vectors for each finger posture to the nearest $2.5 \mathrm{~mm}$ value, the calculated fingertip positions, orientations, and FADs were organized to a 3D grid of points with a mesh size of $2.5 \mathrm{~mm}$.

\subsubsection{Redundancy weighting}

The grid was assigned a color mapping related to the number of unique finger postures that resulted in the fingertip pad reaching each grid location. A larger value indicated a higher level of redundancy in finger postures capable of positioning the finger pad at a particular point.

\subsubsection{Orientation weighting}

The Orientation weighting parameter was based on the range of fingertip orientation vectors collected at each mesh point. At each reachable mesh point in the WFS the two orientation vectors that formed the limits of the angular range were identified and the angle between the limiting vectors was calculated. A larger orientation angle indicated a higher level of functional capacity of the finger to orient the fingertip within the WFS at that location.

\subsubsection{Range of FADs}

The Range of FADs was calculated to be a value that represented the maximum angular range of FAD vectors accumulated at each grid location. Each FAD corresponded to a flexion movement of one of the joints of the finger (MCP, PIP, and DIP) and represented the direction in which a grasping or button actuation force could be generated at the fingertip pad. A larger FAD angle indicated a higher range of FADs and that was considered a larger functional ability as forces could be applied in a greater variety of directions (Fig. 1).

\subsection{Development of group WFS models}

Group WFS models were calculated by merging the WFS models from the individuals in the Healthy and RFA groups and represented the number of the participants out of each group that could reach the same points in 3D space. The individual models were merged by first determining the average position of each $\mathrm{MCP}$ joint of the individuals in each group. Then the WFS models for each finger were translated in the radial-ulnar and proximaldistal directions such that the individual MCP location was moved 
to the group-average MCP location. Finally, the reachable grid points were weighted by the number of individuals from the group that were able to reach each point.

\subsection{Auto-injector}

Auto-injectors are devices that are commonly used by patients to self-inject medicines such as epinephrine and arthritis medications. The auto-injector was chosen for the sample object in this research because it is a simply-modeled cylindrical object that is held and actuated with one hand by people with varying ranges of functionality. Three commercially available auto-injectors are shown in Fig. 2.

The auto-injector was modeled in MATLAB (The Mathworks Inc.; Natick, Massachusetts) as a cylindrical surface mesh of discrete points spaced approximately $2.5 \mathrm{~mm}$ apart. Each mesh point was then representative of a surface area of $6.25 \mathrm{~mm}^{2}$. Five cylinders were modeled with diameters of $20,30,40,50$, and $60 \mathrm{~mm}$. The dimensions were chosen to encompass the range of diameters in the commercially available auto-injectors. The cylinder models were positioned in the 3D space of the WFS model at the proximal-distal level of the MCP joints with their long axes parallel to the line between the second and fifth MCP joints. The long axis of each cylinder was offset from the line between the joint centers of the

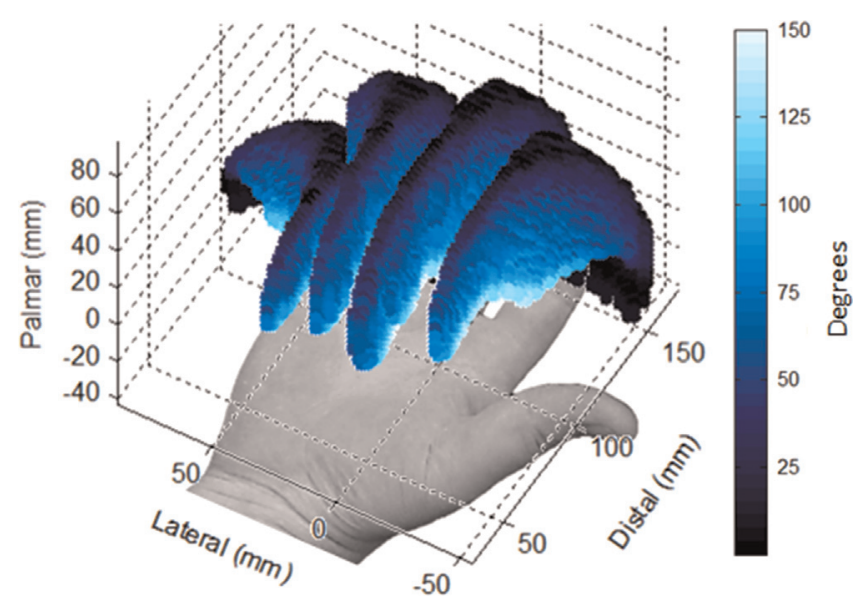

Fig. 1. Three dimensional representation of the WFS model showing the FAD weighting for the index through little fingers with respect to the orientation of the hand. Darker colors indicate lower measures of functionality at a reachable point, lighter colors indicate higher levels of functionality. (For interpretation of the references to color in this figure legend, the reader is referred to the web version of this article.)

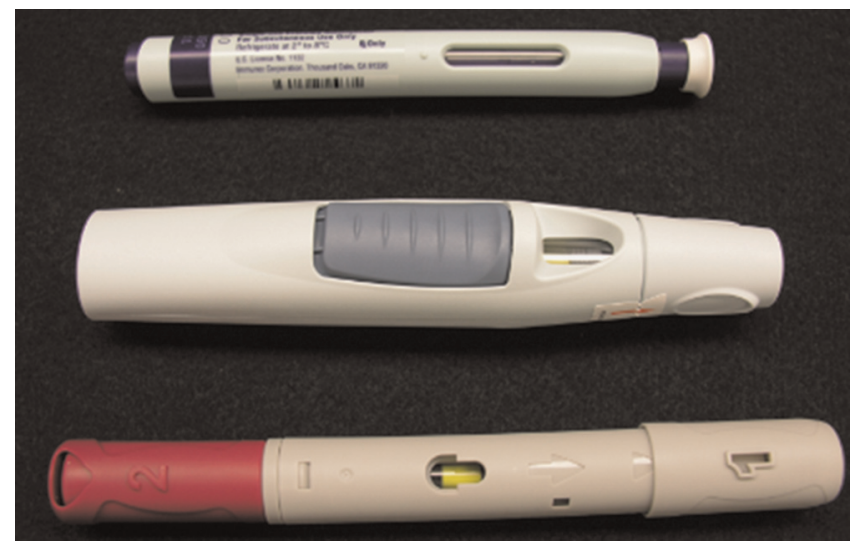

Fig. 2. Three commercially available auto-injectors. Note the auto-injectors vary in diameter and button placement. second and fifth MCP joints in the palmar direction the distance of half the measured MCP thickness plus the radius of the cylinder such that the surface of the cylinder was flush with the palm. To avoid including points that would be physically reachable by the fingers, but not feasible (i.e. the fingers cannot travel through the cylinder and reach the portion of the cylinder in contact with the palm), only surface points on the top half of the cylinder were included in the analysis.

\subsection{Mapping the WFS to the object}

The WFS models were mapped to the surface points of the cylinder to determine where and how each fingertip could interact with the cylinder surface. This was achieved by first merging the WFS model data and the cylinder model surface points in the same space. The WFS point closest to each surface point was identified by rounding the position coordinates of the surface point to the nearest $2.5 \mathrm{~mm}$ increment (the mesh size of the WFS grid). The WFS weighting values from the closest grid point were assigned to that surface point, Fig. 3. The mapping of the WFS onto the cylinder was evaluated for all fingers except the thumb; the thumb pad will not be used to grasp but rather to actuate the firing of the device.

\subsection{Design variation and analysis}

\subsubsection{Individual WFS models}

The WFS model was mapped to the surface of the cylinders for all five diameters for all 43 individuals. Each cylinder diameter was evaluated using the Redundancy weighting, the Orientation weighting, and the FAD weighting. The WFS model mappings were evaluated qualitatively by identifying the shape and size of reachable areas and the patterns of highest and lowest functionality for each weighting parameter. The WFS model mappings were summarized quantitatively using the (1) maximum values of each of the functional weightings that were mapped to the surface points and (2) a summation of the maximum weighting values for each of the four fingers. This last summary parameter provided an overall view of the abilities of all fingers of the hand to interact with the surface of the cylinder.

\subsubsection{Group WFS models}

The two group models were mapped to the five cylinder diameters to analyze how reachable each diameter was for a particular group. The mappings of the group models represented the number of individuals from each group that could reach the same areas on the surface of the cylinders. The number of mapped points to the surface of each cylinder were summed and multiplied by the surface area each point represented $\left(6.25 \mathrm{~mm}^{2}\right)$ to indicate the total amount of reachable area for each percentage of the group. The data for points reachable by at least $50 \%$ and $75 \%$ of the groups were included for both the Healthy and RFA groups. In addition, the data for points reachable by at least $90 \%$ and all individuals were included for the Healthy group.

\section{Results}

\subsection{Mapping the WFS to a cylindrical surface}

Fig. 4 shows a representative Healthy participant's WFS model mapped to a $30 \mathrm{~mm}$ diameter cylinder for all three weighting parameters. The weightings each mapped to the cylinder in unique ways as shown by the distribution of light and dark colors mapped to the surface points. The Redundancy weighting mapped the highest values to the top of the cylinder positioned furthest away from the palm for each finger. The Orientation weighting mapped 

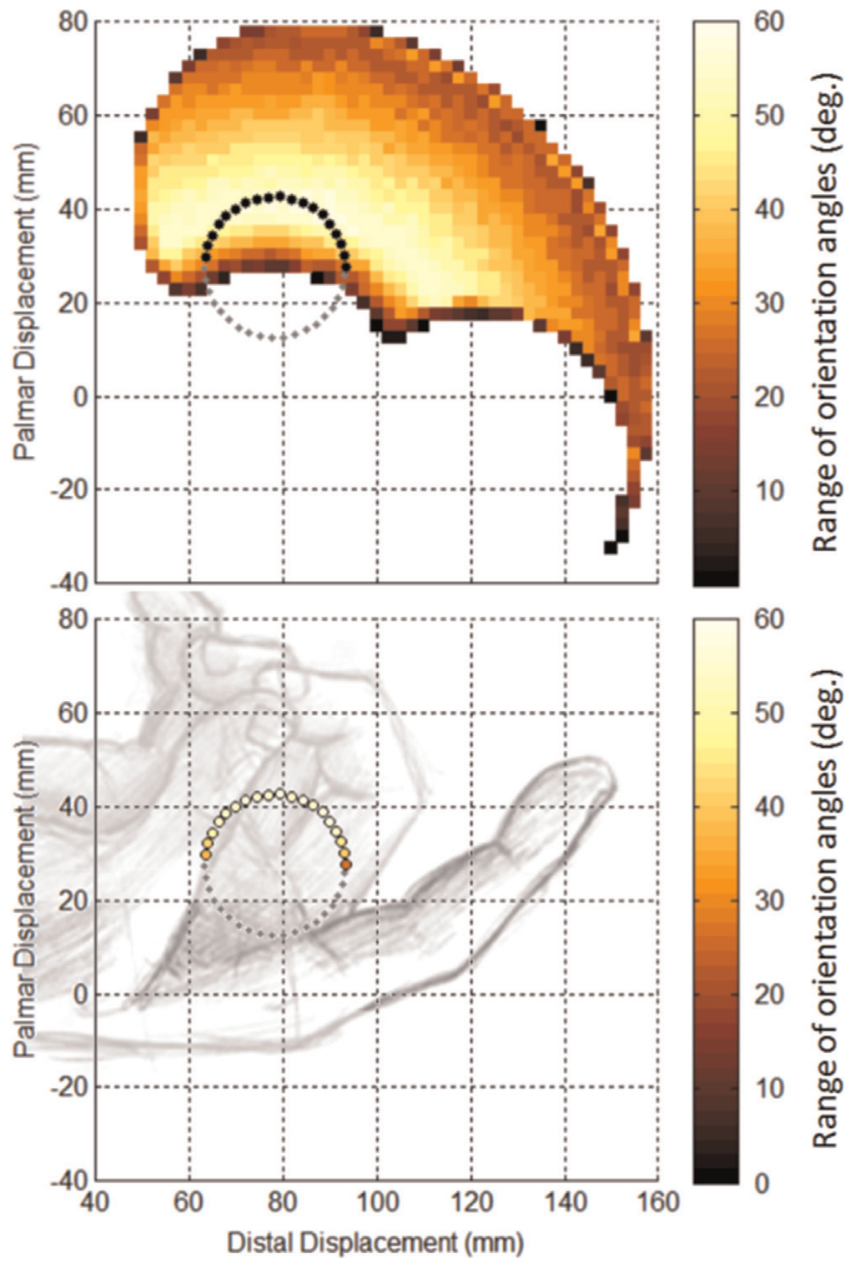

Fig. 3. (Top) Sagittal plane view of the WFS model and the cylinder profile (points in black). Solid gray dots indicate the bottom half of the cylinder. The WFS model is shown for the Orientation weighting with dark orange representing low angular ranges and the lighter yellows representing high angular ranges. (Bottom) WFS values mapped to the surface points of the cylinder model with hand shown for reference. (For interpretation of the references to color in this figure legend, the reader is referred to the web version of this article.)

the areas of highest functionality furthest away from the palm, but in a broader band of points across the top surface. The FAD weighting showed highest levels of functional mapping on the sides of the cylinder for each the fingers.

\subsection{Design variation with the WFS model}

The weightings of the WFS model for the same representative participant are shown in Fig. 5 with the weightings mapped for all five diameters. For the Redundancy weighting, the highest values were positioned on the top of the cylinder for all diameters. For the Orientation weighting, the highest values were at the top of the cylinder for the smaller cylinders (20 and $30 \mathrm{~mm}$ ) and on the distal and proximal sides of the cylinder for the larger cylinders (50 and $60 \mathrm{~mm}$ ). The FAD weighting showed the highest values on the distal and proximal sides of the cylinder for all diameters, but with decreasing total magnitude of the weighting with increasing cylinder size.

Fig. 6 shows the summed maximum weighting values of each hand averaged across each group. The values are shown for the Healthy and RFA groups, with the error bars indicating the standard error for each data point. The Healthy group showed two different optimal diameters for the three weighting parameters. The Redundancy and Orientation weightings both indicated the
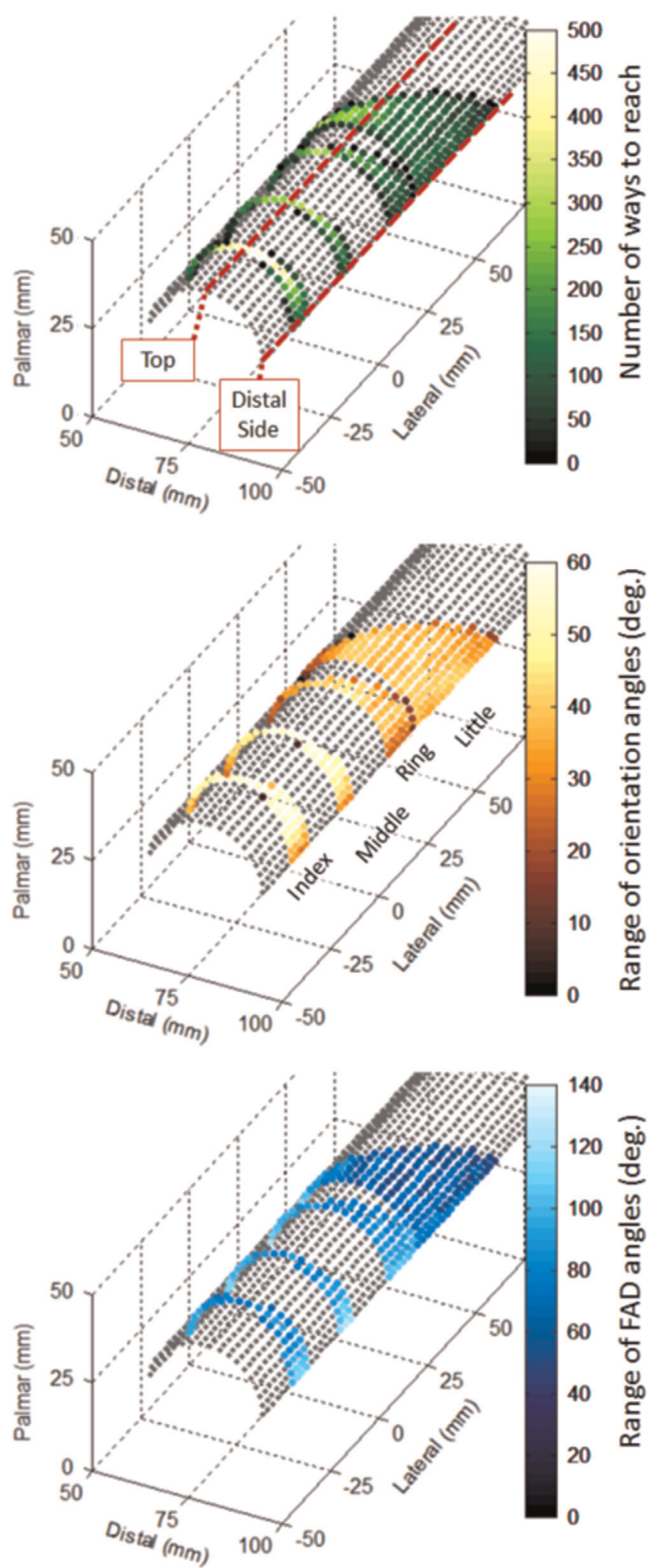

Fig. 4. Three weightings of the WFS model of a single participant mapped to the surface points of the cylinder. (Top) The Redundancy weighting is plotted in shades of green with regions of the cylinder labeled. (Middle) The Orientation angles weighting is plotted in shades of red and orange with the mappings labeled by finger. (Bottom) The $F A D$ weighting is plotted in blue. For all weightings, darker colors represent lower levels of functionality, while lighter colors represent higher levels of functionality. (For interpretation of the references to color in this figure legend, the reader is referred to the web version of this article.) 

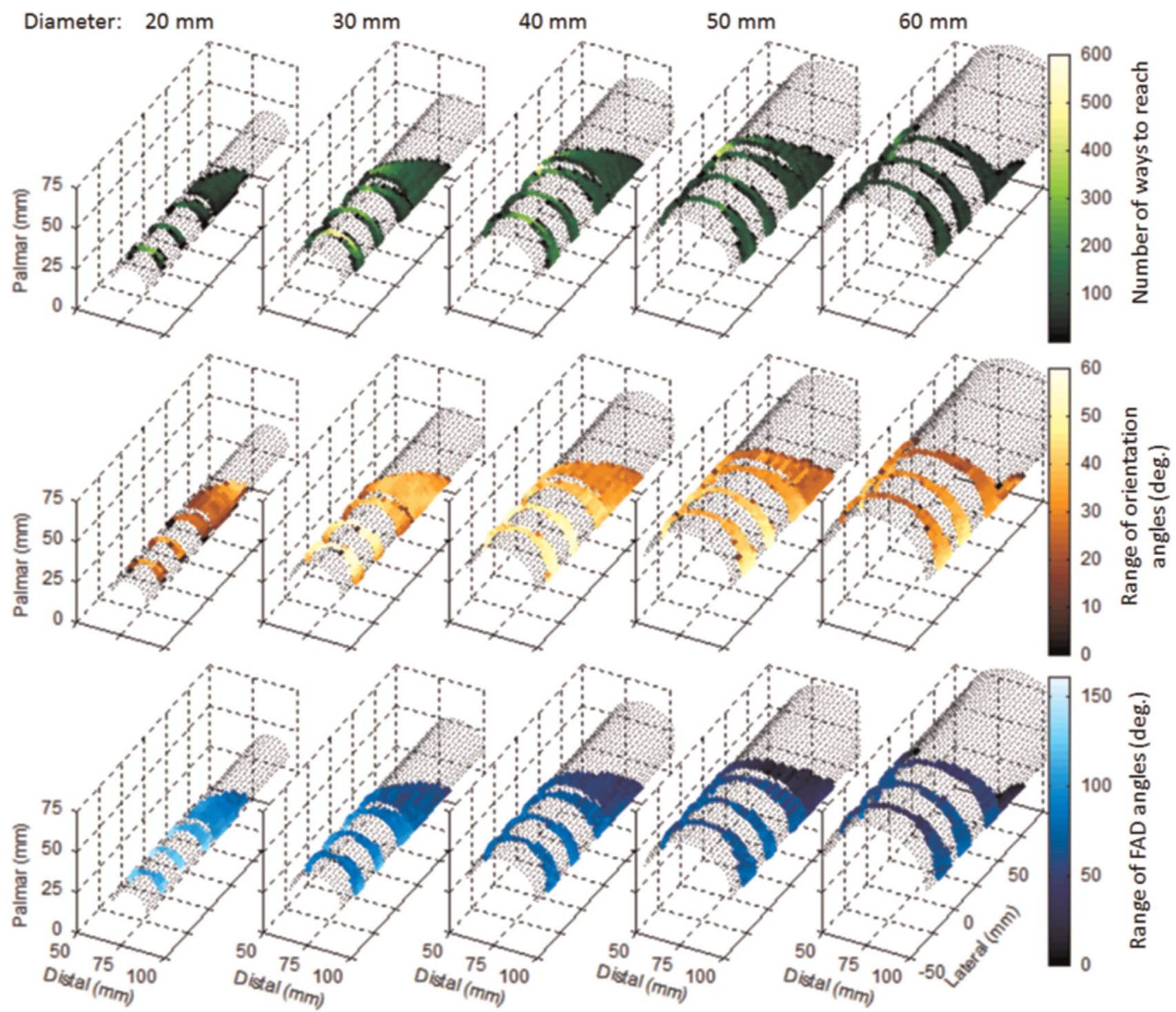

Fig. 5. The three weighting parameter of the WFS model plotted to half cylinders of dimensions ranging from 20 to $60 \mathrm{~mm}$ in diameter.

$40 \mathrm{~mm}$ diameter had the highest cumulative functional weighting. The $F A D$ weighting indicated the $30 \mathrm{~mm}$ diameter had the highest weighting values and $40 \mathrm{~mm}$ with the second highest values.

The RFA group showed three different optimal diameters based on the different weightings. The Redundancy weighting showed the highest weighting at for the $30 \mathrm{~mm}$ cylinder. The Orientation weighting indicated the $50 \mathrm{~mm}$ cylinder had the highest weighting, and the $F A D$ weighting indicated the $40 \mathrm{~mm}$ cylinder as best. Both the Redundancy and Orientation weightings showed $40 \mathrm{~mm}$ as having the second-highest weighting values.

\subsection{Evaluating design inclusion using group models}

Fig. 8 provides summary values for the group models presented in Fig. 7. The cylinders were each analyzed with respect to the percentage of each group that could reach the surface. The values were plotted with respect to the amount of overall area that each percentage of the group could reach on each cylinder with all four fingers. For the Healthy group, the diameter that was most reachable by all of the participants was $40 \mathrm{~mm}$. For the RFA group, no diameters were reachable by all or even $90 \%$ of the group. The highest number of individuals that were able to reach the same point on the surface of a cylinder with any of the fingers was 18 of the 21 participants $(85.7 \%)$. This was true for both the $50 \mathrm{~mm}$ and $60 \mathrm{~mm}$ cylinders. The $40 \mathrm{~mm}$ cylinder showed the largest reachable area when considering whether $50 \%$ or $75 \%$ of the RFA group was able to reach the surface of the cylinder.

\section{Discussion}

\subsection{Mapping the WFS to a cylindrical surface}

A unique aspect of the WFS model is that it presents information about the functional ability of the hand in a 3D frame of reference, as opposed to other single dimensional measures of the hand, such as ROM. Having a 3D space representing hand function allows the WFS model to be mapped to the same space as any 3Dmodeled handheld object, a necessity for design. Currently, no other models of the hand relate differing functional abilities in 3D space to design parameters.

The WFS mapping represents the entire range of possible fingertip placements on the surface of the object. However, when using the WFS for design decisions, the areas of highest functional values should be identified and considered. The ability to identify the areas of highest functionality through the interpretation of colored 3D 

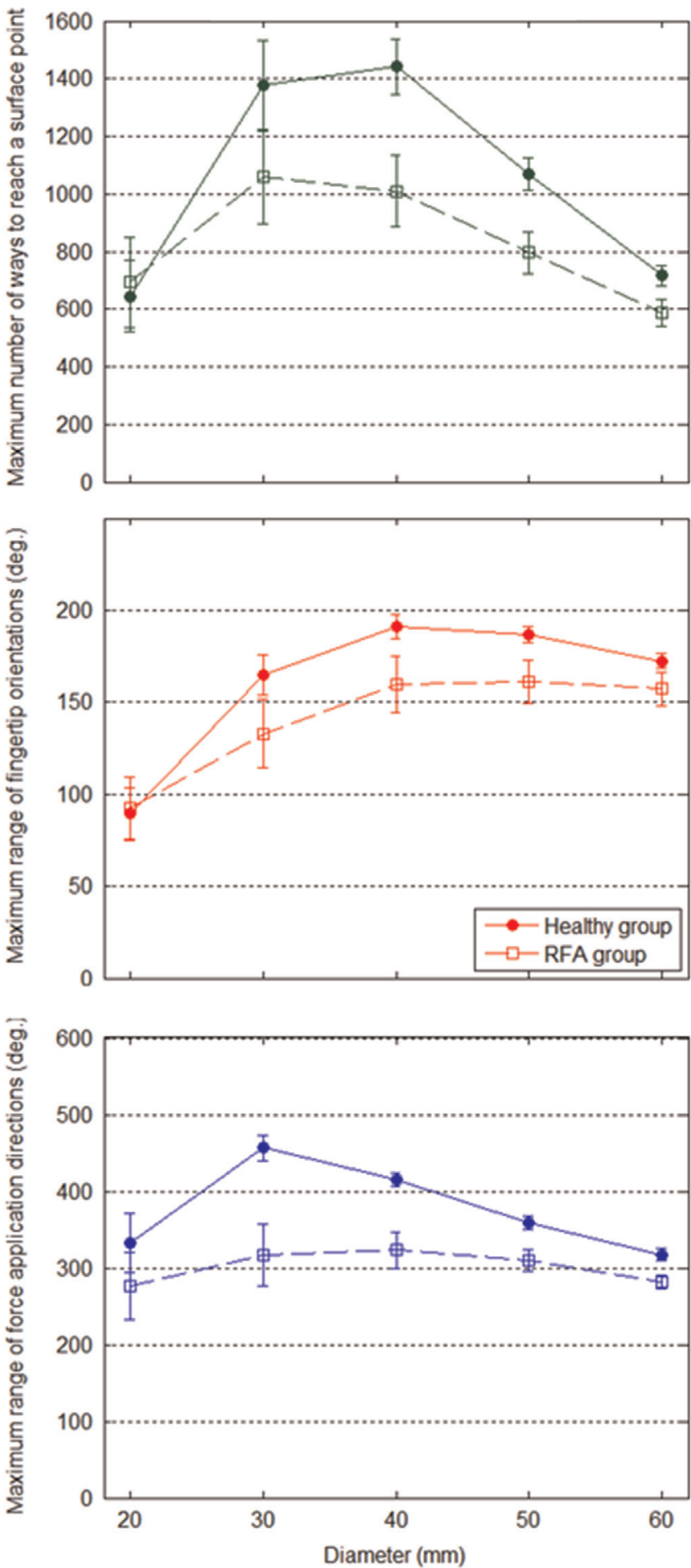

Fig. 6. Summary values of the maximum WFS weightings mapped to the surface of the cylinder plotted by diameter. Data represent the average of summed values of the weightings from all four fingers. Standard error is shown in the error bars for the Healthy and RFA groups. (Top) Redundancy weighting (Middle) Orientation weighting (Bottom) FAD weighting.

mappings is unique to the WFS model mapping and has not been shown with other 3D modeling methods for hand function.

\subsection{Design variation with WFS model}

The mapping of the WFS model to the varying diameters of the cylinder shows that there are quantitative trends that can be leveraged to design objects. The data show that the three functional abilities mapped to the different diameters in unique ways, but no single diameter demonstrated the highest maximum cumulative ability of all four fingers across all functional measures for either group. Therefore, in order to determine a single "best" diameter for the auto-injector based on the WFS mappings, these results should be framed in the context of the needs of the device and the user/device interaction. The user of an auto-injector needs to be able to effectively grab the device, hold it firmly with fingertips flush to the surface, and apply forces in a specific direction. Therefore, the functional weightings should bear a balanced influence in the analysis. Based on this interpretation of the grasp context, it would be recommended for both the Healthy and RFA groups to have an auto-injector with a diameter of $40 \mathrm{~mm}$.

In this way, objective data from humans can be used to support design decisions for handheld objects. This method can be applied to any individual or group of individuals and to any object that can be modeled as a set of surface points. Further, the computational nature of the process means that many design iterations can be tested before expensive physical prototyping or testing of the design occurs.

These mappings also provide a unique perspective to view the motor control of the human hand. Many researchers have investigated the redundancy of the hand as means to understand motor control (Latash et al., 2007; Li et al., 1998; Raghavan et al., 2010; Scholz et al., 2002; Zatsiorsky et al., 2003). The weightings of the WFS model highlight the spatial relationships of kinematic redundancies that exist for a hand to grasp an object. Specific to grasping with the fingertips, Kamper et al. produced work showing that the fingertip travels in stereotypical trajectories when moving to different grasps (Kamper et al., 2003). This would suggest that an individual does not explore all of the reachable space that is available when choosing a grasping strategy, but rather follows rote fingertip paths until contact is made. It is offered here that the fingertips follow the path of greatest kinematic redundancy as presented as weighting values in the WFS model and that this mechanism could be the underlying governing principle behind the stereotypical trajectories. Thus, the area of highest WFS weighting that was mapped to an object would be the most likely placement for the fingertip. Future research will examine the relationships between finger placements of experimentally measured grasps and the weightings of kinematic redundancies of the WFS model when mapped to objects' surfaces.

\subsection{Inclusive design using the group models}

The mappings of the group models on the cylinders were presented to demonstrate the potential of the group models for inclusive design of handheld objects both within a single group and across multiple groups. For both groups, if the goal was to have greater than $75 \%$ of the population capable of reaching the surface, the best diameter was $40 \mathrm{~mm}$. However, for the RFA group, if the goal was to have greater than $85 \%$ of the RFA population contact the device, $50 \mathrm{~mm}$ and $60 \mathrm{~mm}$ diameters were the only two possible diameters with reachable areas. From this, it is recommended that cylinders designed to be grasped in a power grasp by RFA individuals be at least $40 \mathrm{~mm}$ in diameter.

While developed in different ways, and measuring different outcomes, the trends from these data bear a resemblance to the maximum grip strength data for a power grasp. Research has shown that for a power grasp, the maximum grip strength is achieved at a diameter between 30 and $40 \mathrm{~mm}$ (Edgren et al., 2004; Kong and Lowe, 2005). However, while reproduction of the force magnitude results would require customized measurement fixtures for each object and grasp, the only additional input that would be required for reproduction of the results from this WFS mapping would be digital 3D-models of the objects. 

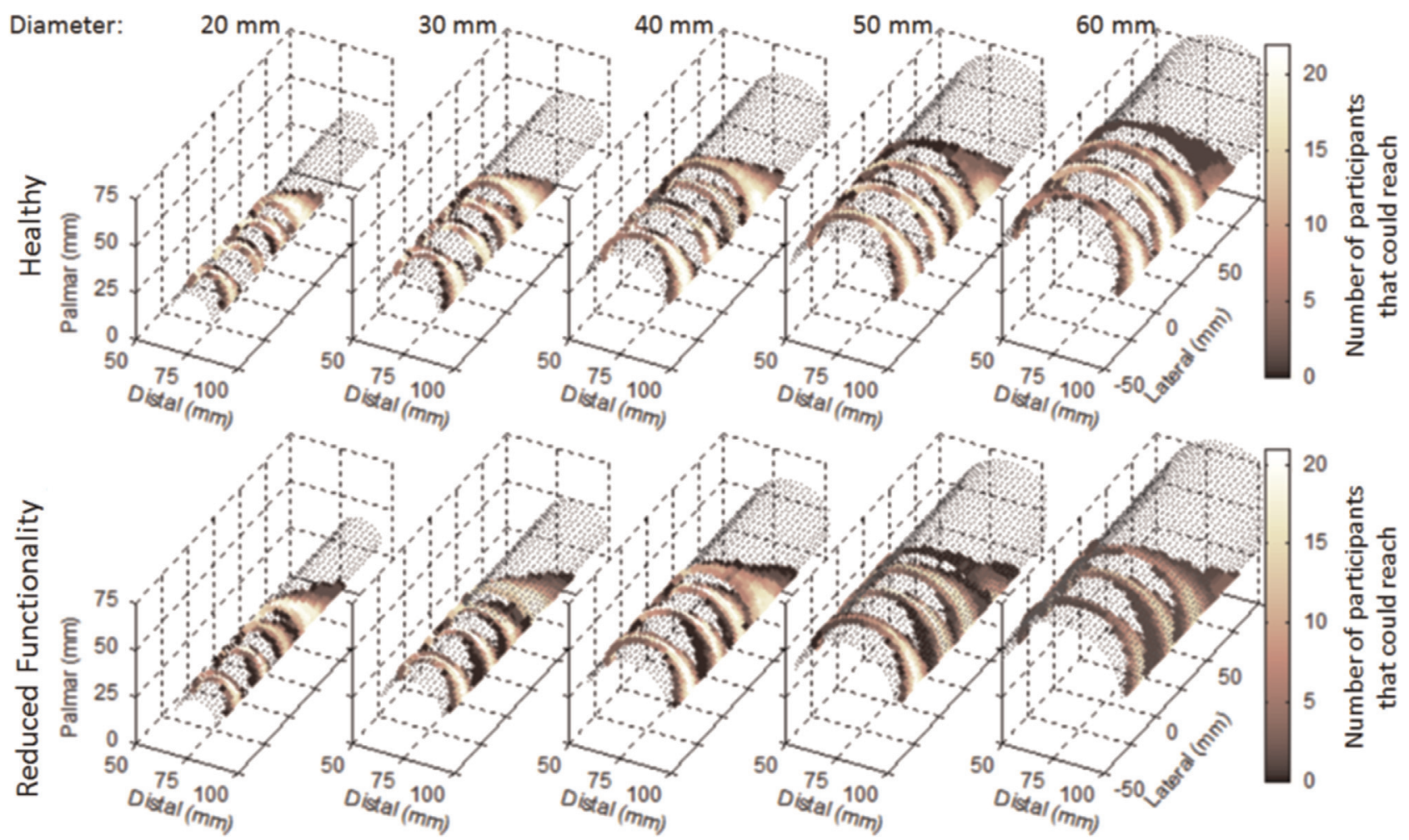

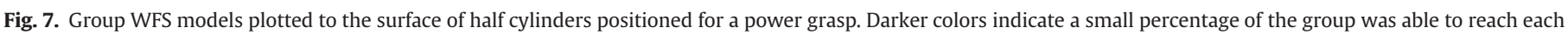

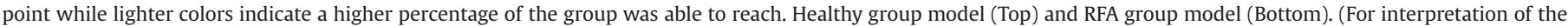
references to color in this figure legend, the reader is referred to the web version of this article.)
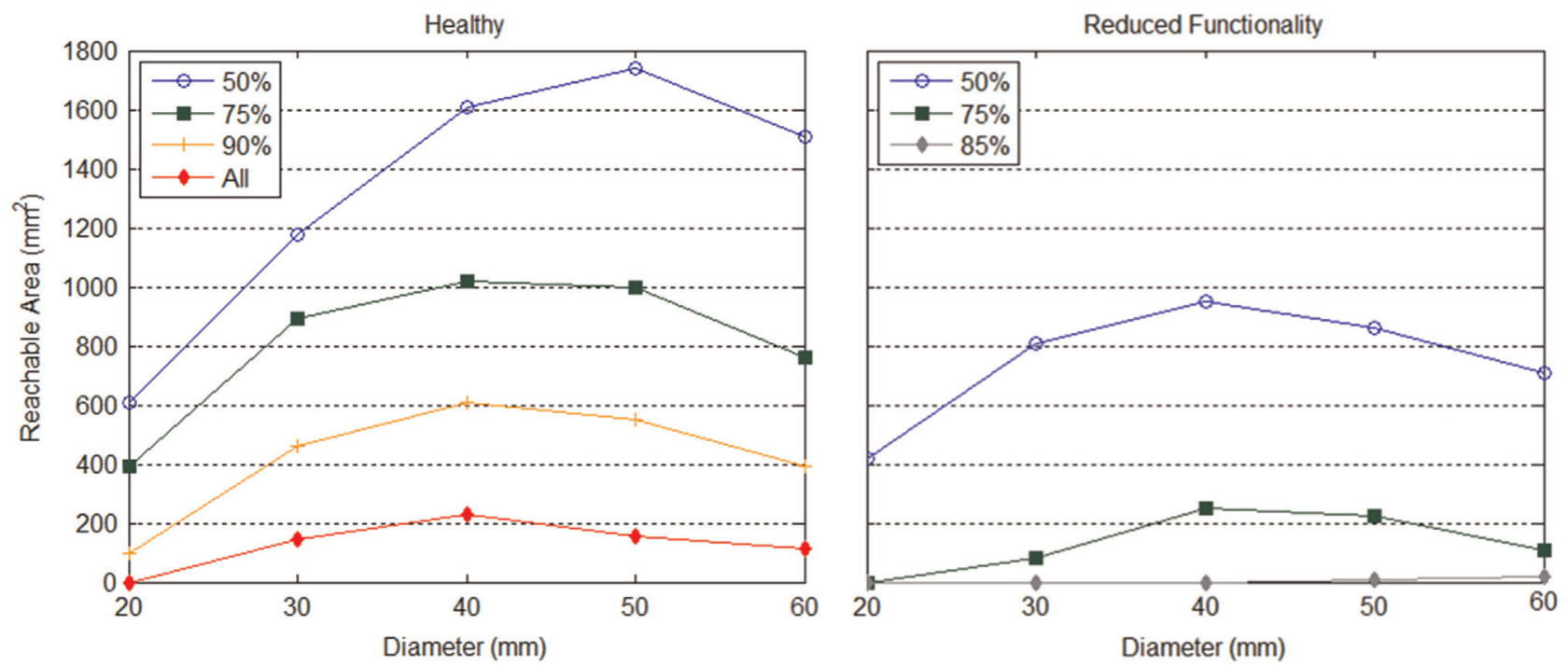

Fig. 8. Summary values of the total amount of reachable area on each half cylinder as plotted by the percentage of each group that could reach the points.

\subsection{Limitations}

There are a few constraints to this mapping process that should be considered during future use. First, finger-finger and palm-object interactions were not accounted for in the mapping process. For example, inter-finger interactions, such as enslaving (Kim et al., 2008; Li et al., 2003), mean that the positions and force capabilities of the fingers are not independent as has been assumed in this model. In addition, while the calculated reachable spaces for the fingers of each hand overlap, no two fingers can occupy the same space at the same time. The result of these assumptions was that the mapping may have been an overestimation of the hand's abilities. Further, the fingertip pads have been simplified to a single point and orientation vector. This underrepresents the palmar surfaces that are available for grasping. Implementation of the surface contours of the palm and fingers, such as those produced by Rogers et al., would improve the accuracy in representation of the whole hands' functional abilities (Rogers et al., 2008). Lastly, the WFS model does not currently account for force magnitude in the weighting values. These force magnitudes will be necessary to more clearly connect with the research being performed on controlling the kinetic redundancy of the hand. These are all issues that the authors will seek to address in future research. 


\section{Conclusions}

This research shows that the data contained in the WFS model can be applied to the surface of a 3D-modeled object, and that the functional mapping can indicate different ways that the fingertip pads can interact with an object. The results further show that variations of a single design can be evaluated to objectively determine which variation best fits the abilities of an individual's hand or a group of individuals' hands. The WFS model has the potential to be used to computationally test object designs for a variety of different hand sizes and abilities before making physical prototypes. In doing so, the tradeoffs of the different functional weighting parameters can be balanced to best fit the desired user population, task, and grasp of the object. This research also shows that the group models developed from the compilation of individual WFS models can be used to determine what percentage of the group will be able to theoretically grasp and touch an object at specific points on the surface. For the example case of the autoinjector these group models showed that $40 \mathrm{~mm}$ was the most reachable diameter of cylinder for a power grasp.

In total, the ability to compare kinematic measures of hand function across variations in handheld devices allows for design creations that are inclusive for the greatest number of individuals across all levels of hand function. Specifically for the RFA population, this can be used to design handheld objects so that people with a reduction in functionality due to arthritis can use the objects needed for maintaining independence.

\section{Conflict of interest statement}

The authors have no financial or personal conflicts of interest to report relevant to development or publication of this research.

\section{Funding sources}

This research was partially funded by the Pearl J. Aldrich Foundation (F5017), the ASHT Founder's Grant (135206), and the Michigan State University College of Engineering Department of Mechanical Engineering. The role of the sponsors was limited to funding the research and did not include influence on the study design; collection, analysis or interpretation of the data; writing of the manuscript; or the decision to submit the manuscript for publication.

\section{Acknowledgments}

The authors would like to thank the Pearl J. Aldrich Foundation and the American Society of Hand Therapists (ASHT) for their financial support. Special thanks go to Josh Drost, Ben Yancho, Anand Saripalli, Eric Waldron, Pan Wu, and Jessica Buschman for their help in data collection and analysis.

\section{References}

Covinsky, K.E., Lindquist, K., Dunlop, D.D., Gill, T.M., Yelin, E., 2008. Effect of arthritis in middle age on older-age functioning. J. Am. Geriatr. Soc. 56, 23-28.

Dellhag, B., Bjelle, A., 1999. A five-year followup of hand function and activities of daily living in rheumatoid arthritis patients. Arthritis Care Res. 12, 33-41.

Dias, J.J., Smith, M., Singh, H.P., Ullah, A.S., 2009. The working space of the hand in rheumatoid arthritis: its impact on disability. J. Hand Surg.-Eur. 34E, 465-470.

Dragulescu, D., Perdereau, V., Drouin, M., Ungureanu, L., Menyhardt, K., 2007. 3D active workspace of human hand anatomical model. Biomed. Eng. Online 6, 15.

Edgren, C.S., Radwin, R.G., Irwin, C.B., 2004. Grip force vectors for varying handle diameters and hand sizes. Hum. Factors J. Hum. Factors Ergon. Soc. 46, 244-251.

Greiner, T., 1991. Hand Anthropometry of US Army Personnel. Security Technical Report p. 434.

Helmick, C.G., Felson, D.T., Lawrence, R.C., Gabriel, S., Hirsch, R., Kwoh, C.K., Liang M.H., Kremers, H.M., Mayes, M.D., Merkel, P.A., Pillemer, S.R., Reveille, J.D. Stone, J.H., 2008. Estimates of the prevalence of arthritis and other rheumatic conditions in the United States. Part I. Arthritis Rheumatol. 58, 15-25.

Jackson, L., 2001. Non-fatal occupational injuries and illnesses treated in hospital emergency departments in the United States. Inj. Prev. 7, i21-i26.

Johnson, R., Yang, J., Long, J., 2010. Finger reach envelope using the marching cubes method. J. Hum. Factors Model. Simul. 1, 321-338.

Kamper, D.G., Cruz, E.G., Siegel, M.P., 2003. Stereotypical fingertip trajectories during grasp. J. Neurophysiol. 90, 3702-3710.

Kim, S.W., Shim, J.K., Zatsiorsky, V.M., Latash, M.L., 2008. Finger inter-dependence: linking the kinetic and kinematic variables. Hum. Mov. Sci. 27, 408-422.

Kong, Y.-K.K., Lowe, B.D., 2005. Optimal cylindrical handle diameter for grip force tasks. Int. J. Ind. Ergon. 35, 495-507.

Kuo, L.C., Chiu, H.Y., Chang, C.W., Hsu, H.Y., Sun, Y.N., 2009. Functional workspace for precision manipulation between thumb and fingers in normal hands. J. Electromyogr. Kinesiol. 19, 829-839.

Latash, M.L., 2010. Motor synergies and the equilibrium-point hypothesis. Mot. Control 14, 294-322.

Latash, M.L., Scholz, J.P., Schoner, G., 2007. Toward a new theory of motor synergies. Mot. Control 11, 276-308.

Leitkam, S.T., Bush, T., Bix, L., 2013. Determining functional finger capabilities of healthy adults: comparing experimental data to a biomechanical model. J. Biomech. Eng. 136, 1-11.

Leitkam, S.T., Bush, T.R., 2015. Comparison between healthy and reduced hand function using ranges of motion and a weighted fingertip space model. J. Biomech. Eng. 137, 041003.

Li, S., Latash, M.L., Zatsiorsky, V.M., 2003. Finger interaction during multi-finger tasks involving finger addition and removal. Exp. Brain Res. 150, 230-236.

Li, Z.-M.N., Latash, M.L., Newell, K.M., Zatsiorsky, V.M., 1998. Motor redundancy during maximal voluntary contraction in four-finger tasks. Exp. Brain Res. 122 $71-78$.

Luckhaupt, S., Dahlhamer, J., 2013. Prevalence and work- relatedness of carpa tunnel syndrome in the working population, United States, 2010 National Health Interview Survey. Am. J. Ind. Med. 56, 615-624.

Raghavan, P., Santello, M., Gordon, A.M., Krakauer, J.W., 2010. Compensatory motor control after stroke: an alternative joint strategy for object-dependent shaping of hand posture. J. Neurophysiol. 103, 3034-3043.

Ranganathan, V.K., Siemionow, V., Sahgal, V., Yue, G.H., 2001. Effects of aging on hand function. J. Am. Geriatr. Soc. 49, 1478-1484.

Roger, V.L., Go, A.S., Lloyd-Jones, D.M., Benjamin, E.J., Berry, J.D., Borden, W.B., Bravata, D.M., Dai, S., Ford, E.S., Fox, C.S., Fullerton, H.J., Gillespie, C., Hailpern, S.M., Heit, J.A., Howard, V.J., Kissela, B.M., Kittner, S.J., Lackland, D.T. Lichtman, J.H., Lisabeth, L.D., Makuc, D.M., Marcus, G.M., Marelli, A., Matchar, D.B., Moy, C. S., Mozaffarian, D., Mussolino, M.E., Nichol, G., Paynter, N.P., Soliman, E.Z., Sorlie, P.D., Sotoodehnia, N., Turan, T.N., Virani, S.S., Wong, N.D., Woo, D., Turner M.B., 2012. Heart disease and stroke statistics-2012 update: a report from the American Heart Association. Circulation 125, e2-e220.

Rogers, M.S., Barr, A.B., Kasemsontitum, B., Rempel, D.M., 2008. A three-dimensional anthropometric solid model of the hand based on landmark measurements. Ergonomics 51, 511-526.

Scholz, J.P., Danion, F., Latash, M.L., Schoner, G., SchoÈner, G., 2002. Understanding finger coordination through analysis of the structure of force variability. Biol. Cybern. 86, 29-39.

Visser, J., Latash, M., Kang, N., 2002. The development of synergies. J. Sport Exerc. Psychol. 24, 129.

Zatsiorsky, V.M., Gao, F., Latash, M.L., 2003. Prehension synergies: effects of object geometry and prescribed torques. Exp. Brain Res. 148, 77-87.

Zatsiorsky, V.M., Li, Z.M., Latash, M.L., 1998. Coordinated force production in multifinger tasks: finger interaction and neural network modeling. Biol. Cybern. 79, 139-150. 